tion errors of the difference method. Moreover, any error introduced in the numerical solution of $y^{\prime}=15 \exp [15 t]$ will be damped by exp $[-14 t]$ in the substitution into $\phi(y, t)=y \exp [-14 t]$.

Tables 1, 2, 3, and 4 give comparisons of relative errors in the numerical solution of $x^{\prime}=15 e^{t}-14 x$ obtained by direct integration, versus the solution obtained by using the alternate equation. The method used is Adams-Bashforth 16th order predictor and Adams-Moulton 15th order corrector. The region of numerical stability (for one application of the corrector) is $-.007 \leqq \bar{h} \leqq .011$. The tables display results using step sizes that caused $\bar{h}$ to lie both inside (Table 1 ) and outside (Tables 2, 3, 4) of the stability region for the direct integration. All integrations connected with the solution using the alternate equation are within the stability region.

Acknowledgment. The authors wish to thank the referees, for their most helpful suggestions and comments, and Mr. John Engvall, for his supporting computer program.

1. R. L. Crane \& R. W. Klopfenstein, "A predictor-corrector algorithm with an increased range of absolute stability," J. Assoc. Comput. Mach., v. 12, 1965, pp. 227-241.

2. L. Fox (Editor), Numerical Solution of Ordinary and Partial Differential Equations, Addison-Wesley, Reading, Mass., 1962, pp. 52-55. MR 26 *4488.

\title{
Some Fourth Order Multipoint Iterative Methods for Solving Equations
}

\author{
By P. Jarratt
}

1. Introduction. Multipoint iterative methods find new approximations to a zero of a function $f(x)$ by sampling $f$ and sometimes its derivatives at each iteration at a number of values of $x$. Although they have not been much used in practice, one interesting class of formulae, investigated by Traub [1, pp. 197-204] is computationally attractive in problems where the evaluation of $f^{\prime}(x)$ is rapid compared with $f(x)$. Such cases arise, for example, when $f(x)$ is defined by an integral. Traub showed that for iterative formulae of the type

$$
\begin{gathered}
x_{n+1}=x_{n}-a_{1} w_{1}\left(x_{n}\right)-a_{2} w_{2}\left(x_{n}\right) \text { where } \\
w_{1}(x)=f(x) / f^{\prime}(x), \quad w_{2}(x)=\frac{f(x)}{f^{\prime}\left[x+\alpha w_{1}(x)\right]},
\end{gathered}
$$

third order processes costing one evaluation of $f(x)$ and two of $f^{\prime}(x)$ per iteration could be constructed by suitable choices of the parameters $a_{1}, a_{2}$ and $\alpha$. It was not possible, however, to obtain fourth order formulae without increasing the number of derivative evaluations. In this paper, a class of iterative methods of the form 


$$
\begin{aligned}
x_{n+1} & =x_{n}-\phi_{1}\left(x_{n}\right)-\phi_{2}\left(x_{n}\right), \text { where } \\
\phi_{1}(x) & =a_{1} w_{1}(x)+a_{2} w_{2}(x) \text { and } \\
\phi_{2}(x) & =\frac{f(x)}{b_{1} f^{\prime}(x)+b_{2} f^{\prime}\left[x+\alpha w_{1}(x)\right]} \quad \text { is examined }
\end{aligned}
$$

and it is shown that in this case fourth order formulae are available costing one function and two derivative evaluations per iteration.

2. Fourth Order Formulae. In order to study the properties of the iteration (1.2) we assume that $f(x)$ has a simple zero at $x=\theta$ and we define the error $\epsilon_{n}$ of the $n$th approximation by $x_{n}=\epsilon_{n}+\theta$. Using now the Taylor expansions of $f\left(x_{n}\right)$ and $f^{\prime}\left(x_{n}\right)$ about $\theta$ we have

$$
w_{1}\left(x_{n}\right)=\epsilon_{n}-\frac{c_{2}}{c_{1}} \epsilon_{n}{ }^{2}+2\left(\frac{c_{2}{ }^{2}}{c_{1}{ }^{2}}-\frac{c_{3}}{c_{1}}\right) \epsilon_{n}{ }^{3}+O\left[\epsilon_{n}{ }^{4}\right]
$$

and hence

$$
\begin{aligned}
w_{2}\left(x_{n}\right) & =\epsilon_{n}-\frac{c_{2}}{c_{1}}(1+2 \alpha) \epsilon_{n}{ }^{2} \\
+ & {\left[2 \frac{c_{2}{ }^{2}}{c_{1}{ }^{2}}\left(2 \alpha^{2}+4 \alpha+1\right)-\frac{c_{3}}{c_{1}}\left(3 \alpha^{2}+6 \alpha+2\right)\right] \epsilon_{n}{ }^{3}+O\left[\epsilon_{n}{ }^{4}\right], \text { where } } \\
c_{r} & =\frac{f^{(r)}(\theta)}{r !} \text { and } c_{0}=f(\theta)=0 .
\end{aligned}
$$

From (2.1) and (2.2) we can show that

$$
\begin{aligned}
\phi_{1}\left(x_{n}\right)=\left(a_{1}+a_{2}\right) \epsilon_{n}-\frac{c_{2}}{c_{1}}\left[a_{1}\right. & \left.+a_{2}(1+2 \alpha)\right] \epsilon_{n}{ }^{2} \\
+\left\{2 \frac { c _ { 2 } { } ^ { 2 } } { c _ { 1 } { } ^ { 2 } } \left[a_{1}\right.\right. & \left.+\left(2 \alpha^{2}+4 \alpha+1\right) a_{2}\right] \\
& \left.\quad-\frac{c_{3}}{c_{1}}\left[2 a_{1}+\left(3 a^{2}+6 \alpha+2\right) a_{2}\right]\right\} \epsilon_{n}{ }^{3}+O\left[\epsilon_{n}{ }^{4}\right]
\end{aligned}
$$

and

$$
\begin{aligned}
& \phi_{2}\left(x_{n}\right)=\frac{c_{1}}{p_{1}} \epsilon_{n}+\left(\begin{array}{l}
c_{2} \\
p_{1}
\end{array}-c_{1} \frac{p_{2}}{p_{1}^{2}}\right) \epsilon_{n}^{2} \\
& +\left[\frac{c_{3}}{p_{1}}+\left(\frac{p_{2}{ }^{2}}{p_{1}{ }^{3}}-\frac{p_{3}}{p_{1}{ }^{2}}\right) c_{1}-\frac{p_{2}}{p_{1}{ }^{2}} c_{2}\right] \epsilon_{n}{ }^{3}+O\left[\epsilon_{n}{ }^{4}\right], \text { where } \\
& p_{1}=c_{1}\left(b_{1}+b_{2}\right), \quad p_{2}=2 c_{2}\left[b_{1}+(1+\alpha) b_{2}\right] \quad \text { and } \\
& p_{3}=3 c_{3} b_{1}+b_{2}\left[3 c_{3}(1+\alpha)^{2}-2 \frac{c_{2}^{2}}{c_{1}} \alpha\right] \text {. }
\end{aligned}
$$

By substituting (2.1)-(2.4) in (1.2) and collecting terms, it can be seen that for 
(1.2) to be fourth order, the following system of equations must be satisfied:

$$
\begin{aligned}
1-a_{1}-a_{2}-\frac{1}{b_{1}+b_{2}} & =0, \\
a_{2}+\frac{b_{2}}{\left(b_{1}+b_{2}\right)^{2}} & =\frac{-1}{2 \alpha}, \\
a_{2}+\frac{b_{2}{ }^{2}}{\left(b_{1}+b_{2}\right)^{3}} & =\frac{1}{2 \alpha^{2}}, \\
a_{2}+\frac{b_{2}}{\left(b_{1}+b_{2}\right)^{2}} & =\frac{1}{3 \alpha^{2}} .
\end{aligned}
$$

This is a set of four equations for the five free parameters $a_{1}, a_{2}, b_{1}, b_{2}$ and $\alpha$,but for consistency we have immediately from the second and last equations that $\alpha=-\frac{2}{3}$. The system (2.5) is hence reduced to

$$
\begin{aligned}
a_{1}+a_{2}+\frac{1}{b_{1}+b_{2}} & =1 \\
a_{2}+\frac{b_{2}}{\left(b_{1}+b_{2}\right)^{2}} & =\frac{3}{4}, \\
a_{2}+\frac{b_{2}{ }^{2}}{\left(b_{1}+b_{2}\right)^{3}} & =\frac{9}{8} .
\end{aligned}
$$

The value $\alpha=-\frac{2}{3}$ is fortunately good for our purposes since it implies that our second sample point, $x_{n}+\alpha w_{1}\left(x_{n}\right)$, is in most cases nearer to the zero than $x_{n}$.

In solving the system (2.6) it is convenient to eliminate $b_{1}$ by writing $b_{2} /\left(b_{1}+b_{2}\right)=\theta$, and for $\theta \neq 0, \theta \neq 1$, the general solution in terms of $\theta$ is then

$$
a_{1}=\frac{1}{4}\left(1+\frac{3}{2 \theta}\right), \quad a_{2}=\frac{3}{4}\left(1-\frac{1}{2(\theta-1)}\right) \quad \text { and } \quad b_{2}=\frac{8 \theta^{2}}{3}(\theta-1)
$$

The special case $\theta=1$ implies $b_{1}=0$ while $\theta=0$ gives $b_{2}=0$. In both instances (1.2) degenerates to (1.1). More generally, with $\theta \neq 0, \theta \neq 1$, we can construct a class of fourth order formulae requiring only one function and two derivative evaluations per iteration by assigning numeric values to $\theta$. Appropriate values of $\theta$ to choose might be those such that the form of (1.2) is simplified. Thus $\theta=-\frac{3}{2}$ gives us $a_{1}=0$, the values of the other parameters then being $a_{2}=\frac{9}{10}, b_{1}=25$, $b_{2}=-15$, while $\theta=\frac{3}{2}$ leads to $a_{1}=\frac{1}{2}, a_{2}=0, b_{1}=-1$ and $b_{2}=3$. This last solution gives the iteration (1.2) the particularly simple form

$$
x_{n+1}=x_{n}-\frac{1}{2} w_{1}\left(x_{n}\right)+\frac{f\left(x_{n}\right)}{f^{\prime}\left(x_{n}\right)-3 f^{\prime}\left[x_{n}-\frac{2}{3} w_{1}\left(x_{n}\right)\right]}
$$

A further suitable choice of parameters which reduces the possibility of cancellation in the denominator of $\phi_{2}$ is obtained by setting $b_{1}=b_{2}$, that is by taking $\theta=\frac{1}{2}$. This gives rise to the solution $a_{1}=1, a_{2}=\frac{3}{2}, b_{1}=b_{2}=-\frac{1}{3}$ and (1.2) now takes the form

$$
x_{n+1}=x_{n}-w_{1}\left(x_{n}\right)-\frac{3}{2} w_{2}\left(x_{n}\right)+\frac{3 f\left(x_{n}\right)}{f^{\prime}\left(x_{n}\right)+f^{\prime}\left[x_{n}-\frac{2}{3} w_{1}\left(x_{n}\right)\right]} .
$$


The asymptotic error constant, i.e. the coefficient of $\epsilon_{n}{ }^{4}$ in the expansion of (1.2) can be obtained by retaining terms of $O\left[\epsilon_{n}{ }^{4}\right]$ in (2.1)-(2.4) and substituting in (1.2) as before. Using the relations (2.6) to simplify, the value of the asymptotic error constant is

$$
\frac{1}{9}(21-8 \theta) \frac{c_{2}^{3}}{c_{1}^{3}}-\frac{c_{2} c_{3}}{c_{1}{ }^{2}}+\frac{1}{9} \frac{c_{4}}{c_{1}},
$$

where the tedious details of the calculation have been omitted. We see that the value $\theta=\frac{21}{8}$ simplifies this constant by removing the first term. The corresponding values of the parameters are

$$
a_{1}=\frac{11}{28}, \quad a_{2}=\frac{27}{52}, \quad b_{1}=\frac{-1183}{64}, \quad b_{2}=\frac{1911}{64} .
$$

3. Conclusions. Four fourth order iterative formulae for solving equations have been derived which require one function and two derivative evaluations per iteration. It is interesting to note that these formulae form counterparts of the iteration function given by Traub [1, p. $184(8-78)]$ which is of order 4 and uses two values of $f$ and one of $f^{\prime}$ per iteration. The formulae obtained in this paper will be particularly appropriate for use in practical root finding problems where the derivative can be quickly computed compared with the function.

4. Acknowledgment. I am grateful to the referee for a number of valuable suggestions.

Computing Laboratory,

Bradford Institute of Technology,

Bradford, England

1. J. F. Traub, Iterative Methods for the Solution of Equations, Prentice-Hall, Englewood Cliffs, N. J. MR $29 \star 6607$.

\title{
Certification of Parlett's ALGOL Eigenvalue Procedure Eig 3*
}

\author{
By J. M. Varah
}

The ALGOL program given by B. Parlett in [1] was tested on the Burroughs B5500 at Stanford University, in two ways. First, the program was checked for correctness of ALGOL 60 syntax, using a program due to William McKeeman [4]. Second, the program was modified to conform to Burroughs Extended ALGOL [3], roughly as in [2], and tested on several matrices. The following errors were found:

1. On p. 477 , line 30 , if $B$ [1] is zero, a divide by zero may be encountered. If the eigenvalue iterate is real, $B$ [4] $=0$ so that if $B$ [1] is zero, control is transferred

Received November 18, 1965.

* This work was sponsored by the Office of Naval Research under contract Nonr 225(37). 